\title{
A Symposium in Honour of Pierre-Michel Duffieux
}

\author{
Peter Hawkes and Noël Bonnet
}

The subject that today we call Fourier Optics may be said to have been created just fifty years ago, with the appearance of L'Intégrale de Fourier et ses Applications à l'Optique [1], privately printed for its author, P.M. Duffieux, by the printing firm of Oberthur in Rennes. The choice of Rennes for the 1996 annual meeting of the Société Française des Microscopies made it natural to devote a symposium and two days of a training course to the role of the Fourier and related transforms in electron microscopy. The present issue of MMM is a record of much of the material that was presented during those three busy days.

The "Duffieux Symposium" opened with a brief introduction by one of us (PWH), followed by a richly illustrated account of Duffieux and his work by J.-C. Viénot, his successor in the chair of Optics at Besançon, to which Duffieux moved after the war. We hope to publish the material assembled by J.-C. Viénot in a future issue. Here, only the main dates associated with L'intégrale de Fourier... are recapitulated. Duffieux's own account of the early years is reproduced below.

In 1941, Duffieux presented his ideas, which were doubtless as yet far from clear in his own mind, at a meeting of the French Physical Society in Paris. His audience apparently understood very little and Fleury urged Duffieux to write an extended account of his work. The first draft of the text was completed in 1943, the date at the end of the Preface; this draft was reorganized to some extent, as explained in the General Introduction, dated 1944. The published text was not printed until 1946, according to the title page; the copyright date is a year later still. The book remained little known outside the world of French optics until 1959, when M. Born and E. Wolf drew attention to it in their Principles of Optics [2] in terms that obliged serious students of the subject to seek it out: "So far we have been concerned with images of point sources. We shall now describe some general methods, based on the techniques of Fourier transforms, relating to the imaging of extended objects. These methods were developed chiefly by Duffieux...".

A decade later, in 1970, six years before the death of Duffieux, a second edition was published by Masson [3] and an English translation of this appeared in 1983 [4].

The 1946 book was not Duffieux's first publication on the subject. Two papers had appeared in the Annales de Physique [5,6] and a third in the Bulletin de la Société Scientifique de Bretagne [7]. Several later papers deal with other aspects of the subject. Many years later, when the question of naming the unit of spatial frequency (the spatial analogue of the hertz) was debated in Applied Optics, one of us urged the claim of the duffieux: $1 \mathrm{Df}=1 \mathrm{~m}^{-1}$ [8]. The units commission of the BIPM was opposed to the introduction of new named units so the proposal had little chance of succeeding - and moreover, "duffieux" was considered too difficult to pronounce by all but his compatriots!

The role of E. Wolf in bringing Duffieux's work to a wide public has already been mentioned and Professor Wolf was good enough to send a short address to the Duffieux Symposium. 
This is reproduced below, together with an autobiographical text written by Duffieux himself for the Conference "Applications de l'Holographie" held in Besançon in 1970.

The role of the Fourier transform is not, however, confined to image formation; it is also an indispensable tool in image post-processing, whether the techniques employed be analogue or digital. With the aid of the Fourier transform we try to simulate a kind of "reverse path", cancelling some at least of the unwelcome features of the image-forming process along the way with a view to restoring the image function. Several lectures and communications were devoted to this image processing aspect of the transform.

Finally, we draw attention to the fact that the transforms that are proving useful in imagery are not static; new transforms arrive, often leading to new ways of thinking of the familiar ones. The evolution of these transforms was addressed in the final paper, by one of us.

The topics examined in this special issue of MMM thus span many different themes: nearfield microscopy (D. Barchiesi), the properties of image digitizers (P. Bron, J.P. Rolland and D. Thomas), thermal imagery (F. Depasse, N. Trannoy and P. Grossel), the Lippmann process for colour image recording (A. Marraud and J.M Fournier), the role of phase in electron image interpretation (M. Hÿtch), the obsolescence of the Fourier transform (N. Bonnet and P. Vautrot) and the evolution of Fourier optics (P.W. Hawkes).

Although we do not claim that these contributions represent all the domains of application of the Fourier and related transforms in optics, we hope that they are sufficiently diverse to justify the issue and we trust that it will help to keep alive the memory of P.-M. Duffieux and the flash of genius that led to his brilliant discovery of Fourier Optics.

\section{References}

[1] Duffieux P.-M., L'Intégrale de Fourier et ses Applications à l'Optique (privately published, 1946).

[2] Born M. and Wolf E., Principles of Optics (Pergamon, Oxford, 1959).

[3] Duffieux P.-M., L'Intégrale de Fourier et ses Applications à l'Optique (Masson, Paris, 1970).

[4] Duffieux P.M., The Fourier Transform and its Applications to Optics (Wiley, New York and Chichester, 1983).

[5] Duffieux P.-M., Ann. Phys. 14 (1940) 302.

[6] Duffieux P.-M., Ann. Phys. 17 (1942) 209.

[7] Duffieux P.-M., Bull. Soc. Sci. Bretagne 17 (1940) 107.

[8] Hawkes P.W., Appl. Opt. 12 (1973) 2537. 


\title{
Appendix I
}

\section{Comment j'ai pris contact avec la transformation de Fourier $\left({ }^{1}\right)$}

\author{
P. Michel Duffieux (Université de Besançon, Faculté des Sciences, la Bouloie)
}

J'avais un peu étudié la transformation de Fourier à Bordeaux, pendant le premier trimestre de l'année scolaire 1918-1919, au cours d'Analyse Supérieure du Professeur Cousin, à la demande de mon chef militaire, le Professeur Henri Bénard. Celui-ci me fit appliquer les équations de Fourier à la mesure de coefficients de conductibilité thermique par la propagation en régime variable. Ce fut ma première publication scientifique. J'y parlais de Fourier : j'étais visiblement prédestiné, mais quand je le retrouvais 15 ans plus tard, je l'avais complètement oublié. Je fus obligé d'aller préparer l'agrégation à Paris, en octobre 1919 : Bénart [sic] fut nommé à Paris au P.C.B. et à partir d'octobre 1920 je fis de l'Optique à Marseille puis à partir de 1927 à Rennes. Là Fabry m'obtint suffisamment de crédits pour monter le meilleur matériel interférentiel à ondes multiples. Celui dont j'avais eu l'usage à Marseille était instable, les lames petites et de qualité médiocre. En Angleterre, en Allemagne, en France on ne me promettait que des plans à $\lambda / 5$, exceptionnellement $\lambda / 10$. Steibelt, qui venait de quitter Jobin, me promit du $\lambda / 20$. En taillant les lames dans du quartz excellent, avec un anneau de garde pour éviter la doucine des bords, il me fit un couple au $1 / 40^{\mathrm{e}}$ de frange, un second au $1 / 20^{\mathrm{e}}$ de frange, un troisième de qualité internationale qu'il me promit de retoucher. J'avais mis au point une méthode d'établissement de la topographie des lames d'air au $200^{\mathrm{e}}$ de frange dès le début du travail; cette topographie était très compliquée.

Je dus abandonner la suite : en Hollande Ostwald monta une équipe d'un genre tout à fait moderne. Il rassembla une vingtaine de spécialistes, compétents, actifs et coopératifs comme on sait l'être en Hollande. En France... et en province, il fallait renoncer.

Rassurez-vous mon matériel de Rennes était encore bon quand mon élève et successeur Jean Viénot entama ses recherches sur le laser. Mais pour moi, les interférences, c'était fini. Mais en 1934 Fabry m'avait dit : "Ostwald utilise la formule de Van Cittert pour les corrections des défauts de planéité de ses lames d'interféromètre. Je l'ai essayée jadis, elle ne vaut rien". Elle consistait à remplacer le cosinus de la formule des franges:

$$
I(x)=\frac{1-r}{1-2 r \cos x+r^{2}}
$$

par l'approximation classique

$$
\cos x=1-\frac{x^{2}}{2}
$$

La période des franges s'étend de $-\pi$ à $+\pi$, le maximum, "la frange", est centré sur $x=0$ et la formule ainsi corrigée est utilisable sur $30^{\circ}$ ou $40^{\circ}$ autour du maximum. "Cherchez quelque chose qui fasse intervenir toute la période", ajouta Fabry. Je lui garde, pour cette remarque, une reconnaissance et une estime que rien de ce qui nous a séparés depuis n'a pu éteindre.

Il me disait cela au début de 1934. Aux grandes vacances, heureusement assez longues en ce temps-là pour que l'on puisse travailler, aussi bien professeurs et chercheurs qu'élèves, je me mis à l'œuvre. C'était difficile et je commençais par les aberrations des franges de deux ondes du Michelson. Au milieu de novembre, après les examens bretons, qui duraient, j'ai envoyé une note avec deux équations l'une en sinus, l'autre en cosinus, donnant : la première la condition

$\left({ }^{1}\right)$ Reproduced by courtesy of M. Goedgebuhr, the present director of the Laboratoire P.M. Duffieux in Besançon. 
de parallélisme optimum, l'autre la position des plans parfaits que l'on pouvait substituer aux surfaces réelles, avec toutes les corrections accessoires désirables.

Le surlendemain matin, Fabry me téléphona à Rennes : "Qu'est-ce que c'est que ces équations ? ; où les avez-vous trouvées ? Je ne les comprends pas et aucun des opticiens que je connais n'en voudra. Trouvez autre chose, mais ça non ; ça ne passera jamais à la Revue d'Optique".

J'allais dans mon amphithéâtre vide, qui avait un long tableau noir et 36 places assises pour 70 P.C.N. habituels ; les autres s'asseyaient, les jambes pendantes, sur la galerie haute qui courrait sur trois côtés et servait au garçon à ouvrir et fermer mes six fenêtres. J'écrivis mes deux équations sur le tableau, je m'assis en face et je les regardai. Dieudonné vint me voir pour raisons de service. "Qu'est-ce que vous faites de ces équations ? Je m'en suis servi pour étudier les aberrations des franges du Michelson et Fabry me demande où je les ai trouvées et qu'est-ce que c'est. Mais ce sont les équations de la transformation de Fourier, série, et réduites au premier terme !". C'était vrai, et à travers 15 ans d'abandon je revis le cours de Cousin de 1918. Je pardonne à Dieudonné son mépris pour Euclide et son cri de guerre nicéen : "Mort au triangle".

J'eus en janvier un troisième secours. Je m'étais remis à Fourier. Dieudonné m'avait vu en première vitesse et fait passer en seconde, mais en janvier suivant une visite presque banale me mit définitivement en $4^{\mathrm{e}}$ et je n'eus plus qu'à m'occuper moi-même de l'accélérateur.

Un matin où j'étais très libre, je reçus un ingénieur de l'Institut d'Optique qui représentait la maison Mader-Ott. Il me montra des appareils de mathématiques et les catalogues de la maison Mader. Je lui pris tout de suite un très beau planimètre d'Amsler. Il me proposa un dispositif nouveau qui en faisait un analyseur harmonique, plus lent que le Corradi, mais beaucoup moins cher et en réalité plus rapide quand on ne demandait que les premiers harmoniques. Tandis qu'il montait les deux appareils, je feuilletais la notice qu'il m'avait ouverte et j'eus tout de suite une révélation hallucinante. Il y avait sur le catalogue la série type de Fourier :

$$
F(x)=\frac{a_{0}}{2}+a_{1} \cos 2 \pi \frac{x}{p}+a_{2} \cos 2 \pi \frac{2 x}{p}+\ldots
$$

Mais je lui substituais la série bien connue que je venais d'enseigner à mes élèves de licence et que je regardais depuis 2 mois :

$$
I(x)=\frac{1}{2}+r \cos 2 \pi \frac{x}{p}+r^{2} \cos 2 \pi \frac{2 x}{p}+\ldots
$$

Ce mélange des deux séries ne me troubla pas et je me dis : "Si tu multiplies tous les $r^{n}$ par le $a_{n}$, tu pourras représenter par cette série toutes les déformations de franges que tu voudras ; la relation entre $F(x)$ et $I(x)$ est une question de mathématiques pures".

J'achetais immédiatement l'analyseur avec toutes ses roues dentées ; nous fîmes les factures en trois exemplaires dont un sur timbre et le représentant de Mader-Ott (Bavière), qui rentrait à Paris eut l'obligeance de me laisser l'analyseur tout monté sur la table. Il est actuellement à Besançon aux travaux pratiques du certificat de licence.

Quant au catalogue décisif de 1935, je l'ai demandé récemment à M. Bulabois qui a eu longtemps la conduite des travaux pratiques. Il l'avait mis de côté, en ayant fait venir d'autres. Comme je regardais la page de couverture assez sale, mais pas trop, il me dit : "Il a beaucoup servi". C'est ce que je désirais.

J'ai écrit en 1963 dans l'Éducation Nationale : "Il y a eu deux parts dans ma vie : j'ai d'abord cherché ma voie, puis un jour, comme cela, brusquement, je l'ai trouvée. J'ai eu quelques secondes pour choisir, j'ai choisi et depuis cet instant-là, je travaille toujours dans la même direction". C'est à la lecture de ce catalogue que je faisais allusion. 
Les $a_{n}$ constituent une fonction ponctuée $f_{0}(u), u$ étant la fréquence courante et les $r^{n}$ une autre fonction ponctuée que je désigne par $i_{0}(u)$. Je connaissais donc la deuxième équation de la convolution

$$
f_{0}(u) \cdot i_{0}(u)=f_{0}^{\prime}(u)
$$

mais je n'avais aucune idée de la première

$$
F(x) \times I(x)=f^{\prime}(x)
$$

J'avais trop peu l'habitude de ce type de calcul pour me tirer vite d'affaire. J'étais d'autre part engagé dans des travaux de spectroscopie avec mon collaborateur Léon Grillet. Je fis donc beaucoup de courbes, d'intégrations graphiques et mécaniques, mais deux ans après j'avais compris. Un an de plus pour trouver la fonction $I(x)$ des franges en ondes multiples et en 1938 j'envoyais, sur sa demande, à M. Guadet, Directeur de la Revue d'Optique, un article qu'il publia. J'avais mis sur le marché la dissipation homogène ; devenue depuis la convolution, qui est un nom beaucoup plus joli et qui fait penser aux liserons de l'été.

Mais je dus cesser toute relation avec Charles Fabry. Il me restait heureusement Aimé Cotton qui me suivait depuis l'École Normale.

La dissipation homogène m'ouvrait directement le rôle de la diffraction dans les images. Je commençais naturellement par les images incohérentes, ou plutôt d'objets incohérents. M. Uffel, jeune candidat à Polytechnique, auquel son père, mobilisé à Rennes pendant la "drôle de guerre", m'avait demandé de donner du travail, m'analysa et dessina les transformées de Fourier des figures de diffraction de la fente et de l'ouverture circulaire. Ces spectres étaient visiblement limités. En 1941, A. Cotton me pria d'exposer ces résultats dans une réunion de la Société de Physique, Place St-Germain des Prés. Personne ne me demanda d'explication, personne ne me discuta. On me laissait tomber.

Mais après la séance, devant des demis, à la brasserie qui est en face des Deux Magots, Fleury et Darmois qui connaissaient les pensées générales m'expliquèrent. Le silence était dû au fait que personne n'avait rien compris ; les opticiens n'avaient qu'une vague idée de Fourier et de ses mathématiques ; on ne les enseignait pas en certificat de licence. Fleury, qui comptait bien prendre la succession de Fabry à la direction de l'Institut d'Optique me demanda d'écrire pour ses ingénieurs un exposé des théories de Fourier utiles à l'Optique.

Ce qui, dans tous les travaux, tous les aperçus que j'eus avant 1944 sur les prolongements optiques de la transformation de Fourier, parut en 46 dans un ouvrage intitulé, en imitation de Norbert Wiener "L'intégrale de Fourier et ses applications à l'Optique". Des parties qui n'avaient pu être incorporées faute d'argent et de papier, parurent en 1945-46 dans les Annales de Physique.

Cotton m'avait dit, en me donnant son opinion sur le manuscrit : en France vous mettrez dix ans pour réussir... à condition que vous reveniez d'Angleterre. M. Maréchal devança, très seul, les dix années prévues, mais enfin j'abandonne la suite de cette histoire au japonais Kubota, à l'australien Steel, aux suédois Ingelstam et Djurle, à l'américain Stroke, à l'anglais H.H. Hopkins qui me ramena ostensiblement en France et m'enleva Jean Viénot pour deux ans afin qu'il le prenne mieux sur place. 


\section{Appendix II}

\section{Message addressed to the Société Française des Microscopies on the occasion of the Duffieux Symposium by Emil Wolf, Wilson Professor of Optical Physics, University of Rochester}

Unlike many other branches of science, the field of optics does not have a comprehensive published history. But when one is eventually written, the name of Pierre-Michel Duffieux will surely be included in a prominent way.

I suspect that Duffieux' career and his many contributions to optics will be reviewed by others at this meeting so I will say just a few words about the highly original and influential contribution which is the occasion for this meeting: namely, the publication of his book L'Intégrale de Fourier et ses Applications à l'Optique. Duffieux wrote the book during the Second World War and it was published privately in 1946, by which time Duffieux had moved from Rennes to Besançon. In 1970, it was republished and brought to the attention of a wider audience by the publishing house Masson in Paris. In 1983, an English translation of it was published by John Wiley in the United States. Thus several decades went by before the book became generally available. In the intervening years, other scientists, notably O. Schade in the USA and H.H. Hopkins in England also made important contributions to this field, but no-one would seriously deny the central role which Duffieux' book played in the creation of a field now generally known as Fourier Optics. Concepts such as "frequency response function", "transmission function" and "transfer function" of an optical system should rightly be always associated with Duffieux' name. These concepts put on a broader foundation some ideas which other eminent scientists such as Ernst Abbe and Lord Rayleigh had conceived much earlier in connection with the resolving power of optical systems.

Apart from optics, Duffieux was knowledgeable and accomplished in other fields, especially in philosophy and music. Like many of his contemporaries and, in fact, most physicists during the past seventy years, Duffieux puzzled over the meaning and interpretation of quantum mechanics. He often talked about one of his conceptual creations (mentioned in the Preface to his book) namely Jumbo, the elephant; he used it sometimes to illustrate the concept of localization of a photon. I do not have a copy of his original drawing but this is my recollection of it:

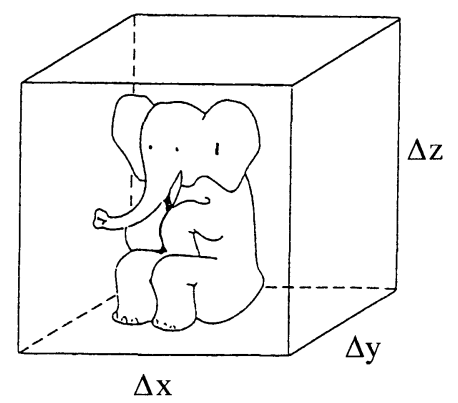

Let me end these brief remarks with the obvious observation that Pierre-Michel Duffieux deserves to be remembered as the true originator of the field of Fourier Optics; and, as I indicated at the beginning of this message, his place in the history of optics seems to be assured.

I wish you a successful symposium! 